remainder of this volume comprises chapters on synthetic systems. That on Surfactants by Kresheck provides a nice summary of micelles and micelleforming molecules; the chapter on Dyestuffs by Duff and Giles emphasises the spectroscopic effects of dye aggregation and stacking interactions, and as such provides a useful and less frequently presented starting point for considerations of the interactions involved in 'stacking' nucleic acids, etc. The final chapter on Synthetic Polymers, by Molyneaux, helps us to organise this vast literature.

In some senses volume 5 , which deals largely with what seem to be totally intractable systems, represents the more unusual of these volumes. It opens with a detailed but well presented chapter on Colloidal Systems, again by Eaglund, which pulls together many aspects of the present state of colloid chemistry. The chapter by Clifford on Water in Capillaries and Thin Films, is, as one might expect, mostly descriptive. It presents, however, one of the most balanced and coherent summaries I have read of two very controversial areas: the status of anomalous water ('polywater'), and of water in cells and tissues. Forslind and Jacobsson use the chapter on Clay-Water Systems in part to provide us a summary of their pointof-view on water structure and watersurface interactions. The remainder of the volume is devoted to chapters on water in very special environments: including Foams and Emulsions (Phillips), Solid Surfaces (Zettlemoyer, Micale and Klier) and Biopolymer Surfaces (Berendsen). Here, as one might expect, the behaviour of water is primarily modulated by the molecular properties of the material comprising the surface or interface at issue, and less by the properties of bulk water.

Overall, Franks and his colleagues have done well by us, providing an appreciation of the ubiquitous effects of water in controlling almost all of life processes, and at the same time providing us with a point at which to approach the various problems of concern to individual investigators. This treatise should be available to all serious research workers concerned with chemical or biological problems in a primarily aqueous world.

Peter H. von Hippel

Erratum. In the review of Lunar Science: A Post-Apollo View (S. R. Taylor) by S. K. Runcorn (Nature, 260, 813, 1976), paragraph 3, line 20 should read: “ . . . implausibleVelikovsky's predictions that . . .".

\section{Crystallinity of macromolecules}

Macromolecular Physics. Vol. 2: Crystal Nucleation, Growth, Annealing. By Bernhard Wunderlich. Pp. $x i+461$. Academic: New York and London, March 1976.) \$46.50; £25.60.

THIS is the second volume in a series so far devoted to phenomena associated with the crystallinity of macromolecules. Volume 1 having dealt with structural aspects, the present volume covers the crystallisation process itself in three consecutive chapters headed by the three subtitles of the book. The book has been long awaited in response to a very real need. This is a work primarily for the active researcher in the field but should also be most useful as a reference book for wider readership in search of information. The book follows no traditional pattern as regards the detailed subject matter or its organisation, and as such is without rival. Its function, as I see it, is intended to be twofold: compilation of knowledge as it exists at present; placing this knowledge in the framework of fundamentals.

As regards my first point, the book is of inestimable value for anybody involved in the subject. Much of the information contained is outside the scope of the usual 'handbook' classification and simply cannot be obtained elsewhere except for the widely scattered original papers. As in volume 1 the Tables are particularly of value in this respect and so is the comprehensive bibliography giving full titles. Also for a book of this size the information content is remarkably up to date.

In addition, the unconventional juxtaposition of an unusually wide range of different subjects in support of particular themes is both informative and stimulating. For example, it contains material on liquid crystals, on macroscopic polymer crystals obtained from polymerisation in the solid state, on simultaneous polymerisation and crystallisation a particularly unique feature of the book), on organic materials, and even on elements forming polymers-all subjects which although relevant, normally do not feature in the standard polymer textbooks.

To achieve the second purpose the author goes back to the fundamentals of phase transitions before his treatment of polymers. Whether these extensive introductions would stand their ground as independent treatises may well be questioned. Even so, they represent a laudable endeavour for a book on polymers. It is a further question how long the present theoretical framework will remain accurate, or even pertinent, as the subject is still in a state of development; there have been changes even since the book went to the press. Even so, the author is to be praised for his courage in investing all this work in a subject still in a state of flux.

Although, as in volume 1 , there is still too much uncritical juxtaposition of facts, there are now certain firm anchor points: altogether a more deliberate attempt has been made to provide critical guidance when presenting conflicting information than in the first volume.

The book is beautifully and carefully produced. As in volume 1, the author has even gone to the trouble of redrawing diagrams to suit his points and to achieve the high aesthetic standards he set for himself.

Altogether it is a welcome and laudable effort.

A. Keller

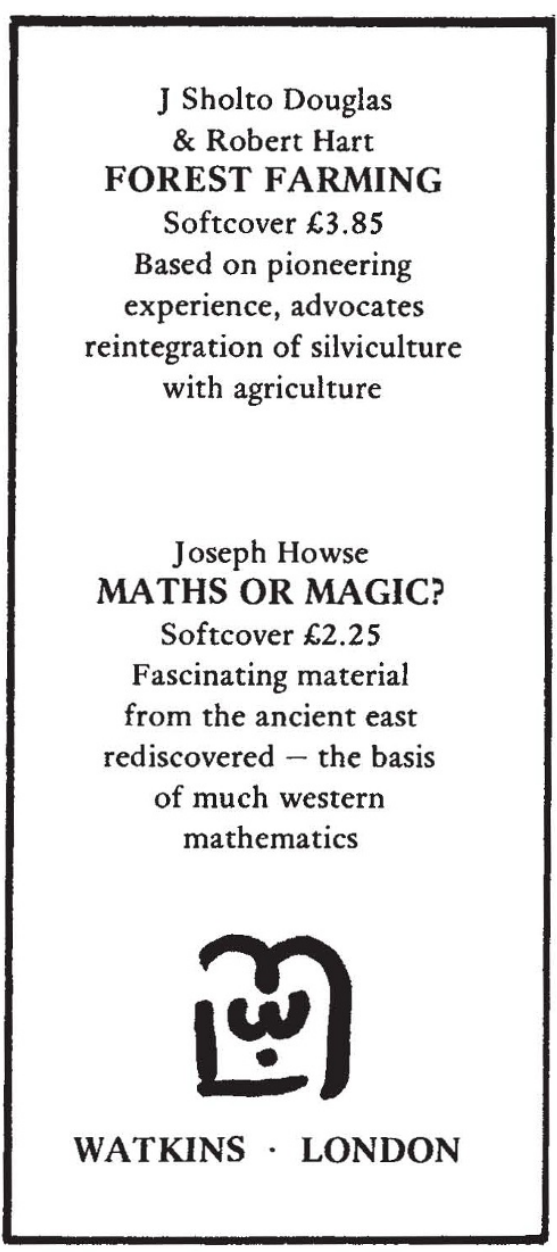

